\title{
Combination of AAV-mediated NUPR1 knockdown and trifluoperazine induces premature senescence in human lung adenocarcinoma A549 cells in nude mice
}

\author{
YANZHE LI ${ }^{1}$, YUEYUAN YIN ${ }^{1}$, JINYI MA ${ }^{1}$, YANAN SUN ${ }^{1}$, RUIMIN ZHOU $^{1}$, BO CUI $^{2}$, \\ YUXUAN ZHANG ${ }^{3}$, JIE YANG ${ }^{1}$, XIAOJIE YAN ${ }^{1,4}$, ZHE LIU ${ }^{1}$ and ZHENYI MA ${ }^{1}$ \\ ${ }^{1}$ Tianjin Key Laboratory of Medical Epigenetics, Key Laboratory of Immune Microenvironment and Disease \\ (Ministry of Education), Department of Immunology, Biochemistry and Molecular Biology, Tianjin Medical University, \\ Tianjin 300070; ${ }^{2}$ Department of Traditional Chinese Medicine, Yangzhou East Hospital, Yangzhou, Jiangsu 225002; \\ ${ }^{3}$ Center for Translational Systems Biology and Neuroscience and Key Laboratory of Integrative Biomedicine \\ for Brain Diseases, Nanjing University of Chinese Medicine, Nanjing, Jiangsu 210023; ${ }^{4}$ State Key \\ Laboratory of Medicinal Chemical Biology, Nankai University, Tianjin 300071, P.R. China
}

Received July 24, 2019; Accepted December 2, 2019

DOI: $10.3892 /$ or.2020.7455

\begin{abstract}
Nuclear protein 1 (NUPR1)/p8, a transcriptional regulator, has the ability to facilitate lung cancer cell survival. Adeno-associated virus (AAV)-based vectors are efficient vehicles for gene transfer and expression. In this study, an AAV-mediated NUPR1 shRNA vector was constructed that effectively inhibited the expression of NUPRI in a tumor xenograft model derived from lung adenocarcinoma A549 cells. Trifluoperazine (TFP), which is an antipsychotic drug, has the ability to bind to NUPR1 and mimic NUPR1 deficiency in cancer cells. It was also found that the combination of TFP and AAV-mediated NUPRI shRNA delivery led to significant tumor growth inhibition in nude mice bearing human lung cancer xenografts. Moreover, AAV-mediated NUPRI shRNA therapy induced premature senescence in vitro and in vivo. Collectively, the findings of this study suggest a putative role for the combination of AAV-NUPRI shRNA and TFP in lung cancer therapy.
\end{abstract}

\section{Introduction}

Lung cancer is responsible for the majority of cancer-related deaths worldwide. The survival rate is related to the stage of

Correspondence to: Dr Zhenyi Ma or Dr Xiaojie Yan, Tianjin Key Laboratory of Medical Epigenetics, Key Laboratory of Immune Microenvironment and Disease (Ministry of Education), Department of Immunology, Biochemistry and Molecular Biology, Tianjin Medical University, 22 Qixiangtai Road, Heping, Tianjin 300070, P.R. China

E-mail: zhyma@tmu.edu.cn

E-mail: xjyan@tmu.edu.cn

Key words: NUPR1/p8, TFP, autophagy, lung cancer, premature senescence lung cancer, and there are regional differences (1). Non-small cell lung cancer (NSCLC) is the main type of lung cancer, which includes adenocarcinoma, squamous cell carcinoma and large cell carcinoma, accounting for $80-85 \%$ of the total number of lung cancer cases. Due to the high malignancy of NSCLC, the irreversible distant metastasis of tumor cells typically occurs prior to the initial diagnosis in the majority of patients $(2,3)$. Therefore, novel therapeutic targets for the successful treatment of lung cancer are urgently required.

Nuclear protein-1 (NUPR1), which is also known as candidate of metastasis-1 (Com1), consists of 82 amino acids, and its structure is highly unstable $(4,5)$. NUPR1 is a transcriptional regulator that can be induced under several cellular stresses, such as hypoxia and starvation (6-8), thereby regulating autophagy through intracellular signaling to counteract adverse situations (9). First, during metastasis, NUPR1 facilitates the adaptation of tumor cells to a new microenvironment and enables them to form new cancer nests (10-12). Survival analysis of patients with medullary thyroid tumors has indicated that NUPR1 is associated with an unfavorable prognosis of patients with thyroid cancer. More specifically, the expression level of NUPR1 is closely related to lymph node metastasis, and $24.8 \%$ of patients with NUPR1 expression have a higher recurrence rate $(13,14)$. Moreover, the downregulation of NUPRI by siRNA has been shown to significantly promote G1 phase cell cycle arrest $(15,16)$ and decrease the proliferation, in vitro soft agar colony formation and tumorigenic ability of NSCLC cells in nude mice (9). Similarly, a high expression of NUPR1 also promotes the proliferation of pancreatic cancer cells (17). Therefore, small compounds or inhibitors that target NUPR 1 for cancer therapy are underdeveloped. For instance, chemicals that inhibit NUPR1 function and mimic NUPR1 deficiency benefit the treatment of pancreatic ductal adenocarcinoma (PDAC) (18). Among these chemicals, trifluoperazine dihydrochloride (TFP) has shown great promise to bind NUPR1 and induce cell growth arrest, mimicking NUPR1 deficiency in pancreatic adenocarcinoma cells (19); however, 
its primary clinical use is as an antipsychotic drug for the treatment of delusional schizophrenia, acute schizophrenic psychosis and chorea (20). Its pharmacological functions involve the blocking of dopamine receptors and functioning as a calmodulin antagonist to bind to calmodulin and interfere with $\mathrm{Ca}^{2+}$-calmodulin interactions (21). However, its efficacy in cancer treatment has only been mentioned in a limited number of studies reporting that TFP inhibits cell invasion and proliferation, and induces cell death in several types of animal models and cancer cell lines (22-25), without addressing the underlying mechanisms or its potential targets.

The authors have previously demonstrated that the downregulation of NUPR 1 induces the formation of autophagic vesicles and cell cycle arrest in NSCLC cells, inducing premature senescence, thereby impairing the proliferation and colony formation ability of NSCLC cells (9). This prompted the exploration of the effects of manipulating NUPRl by shRNA and small compounds on lung cancer progression in vivo.

Adeno-associated virus (AAV)-based vectors lack pathogenicity, but are efficient vehicles for gene delivery, rendering them a potentially useful tool in gene therapy $(26,27)$. In this study, we used a recombinant AAV vector expressing NUPRI shRNA combined with TFP to examine the effect of a NUPR1 'double kill' approach on A549 cells in vitro and in vivo. It was found that TFP combined with NUPRI shRNA using AAV vector delivery enhanced the effects of TFP, impairing autophagy, promoting premature senescence and inhibiting tumor growth in vitro and in vivo. The findings of this study may aid in the development of novel effective therapeutic strategies for lung cancer.

\section{Materials and methods}

Cell lines. A549 and 293AD cells were obtained from the American Type Culture Collection (CCL-185 and CRL-1573, respectively) and were cultured according to the recommended protocols.

Viral particle production. Oligonucleotides encoding shRNA for NUPR1 (GGAGGACCCAGGACAGGATCC) or Firefly luciferase (CGTACGCGGAATACTTCGATT) as a control were ligated into pSUPER.retro.puro, and the fragment containing the $\mathrm{H} 1$ promoter and hairpin sequences was subcloned into the pAAV vector (Cell Biolabs) according to the manufacturer's protocol. The production and purification of AAV were carried out as previously described (28). The AAV titer was quantified by qPCR analysis.

In vitro transgene expression. A549 cells were cultured in complete medium and treated with AAV particles for $48 \mathrm{~h}$. Fluorescence microscopy (magnification, x10; Olympus Corp.) was used to analyze the infection efficiency of AAV particles. The infected cells were collected for the analysis of the protein levels.

Western blot analysis. Whole cell protein extracts in $1 \mathrm{X}$ Laemmli buffer (Bio-Rad, cat. no. 1610737) were resolved through $15 \%$ SDS-PAGE, transferred to a nitrocellulose membrane, and probed with antibodies against human
NUPR1 (cat. no. 1:1,000; Abcam), SNAP25 (cat. no. ab109105; 1:1,000; Abcam), p62 (cat. no. 7695, 1:1,000; Cell Signaling Technology), p16 (cat. no. ab108349, 1:2,000; Abcam), p21 (cat. no. 9665; 1:2,000; Cell Signaling Technology), p27 (cat. no. 9932; 1:2,000; Cell Signaling Technology) and endogenous ACTB (cat. no. A-3853; 1:3,000; Sigma-Aldrich; Merck $\mathrm{KGaA}$ ) as a control for normalization. Peroxidase-conjugated anti-mouse (cat. no. A-10654; 1:5,000; Thermo Fisher Scientific, Inc.) and anti-rabbit IgG (cat. no. 31423; 1:5,000; Thermo Fisher Scientific, Inc.) were used as secondary antibodies. The blots were visualized on a Kodak X-ray film using an enhanced chemiluminescence (ECL) detection substrate (cat. no. 32106, Thermo Fisher Scientific, Inc.). ImageJ software (version 1.0; National Institutes of Health) was used to quantify the intensities of band signals, which were normalized to the ACTB internal controls.

Tumor xenografts. Animal care and surgical procedures were approved by Tianjin Medical University and carried out in accordance with the Institutional Animal Care and Use Committee guidelines (Tianjin Medical University, permission no. SYXK: 2016-0012). A total of 16 male Fox Chase severe combined immunodeficient (SCID) mice were housed at $18^{\circ} \mathrm{C}$ and supplied with water and laboratory chow. The SCID mice were subjected to subcutaneous injections of A549 cells (1.0x10 ${ }^{6}$ cells) in Matrigel (50:50) into the lower flank. Ten days following tumor inoculation, the mice were randomly divided into four groups and received tail vein injections of AAV-iNUPR1, AAV-control $\left(1 \times 10^{11} \mathrm{vg} / \mathrm{mouse}\right), \mathrm{TFP}$, or saline. To evaluate the effect of AAV and TFP treatment on tumor growth, tumor volume was measured at the indicated time points using a scaled ruler. After anesthetized by intraperitoneal injection with $10 \%$ chloral hydrate $(300 \mathrm{mg} / \mathrm{kg}$ body weight), the mice were sacrificed by cervical dislocation. Death was confirmed when the mice did not move, and then tumor tissues were collected and weighed immediately, followed by fixation in buffered formalin for histological and immunohistochemical analyses.

Transmission electron microscopy. Tumor tissue obtained from the animals was washed with PBS and fixed with PBS containing $3 \%$ glutaraldehyde and $2 \%$ paraformaldehyde (cat. nos. 340855 and P6148, respectively; Sigma-Aldrich; Merck KGaA) (pH 7.3) for $2 \mathrm{~h}$ at room temperature. Following fixation, the samples were washed with PBS and post-fixed with $1 \%$ buffered osmium tetroxide (cat. no. 75632, Sigma-Aldrich; Merck $\mathrm{KGaA}$ ) for $30 \mathrm{~min}$ at room temperature and stained en bloc with $1 \%$ uranyl acetate (cat. no. 19481, Ted Pella, Inc.). The samples were dehydrated in increasing concentrations of ethanol, embedded in EMbed 812 medium (cat. no. 14120, Electron Microscopy Sciences) and polymerized at $70^{\circ} \mathrm{C}$ for 2 days. Ultrathin sections were cut using a Leica Ultracut microtome (Leica), stained with uranyl acetate and lead citrate in a Leica EM Stainer and examined using a JEM 1010 transmission electron microscope (JEOL) at an accelerating voltage of $80 \mathrm{kV}$. Digital TEM images were acquired using an AMT Imaging System (Advanced Microscopy Techniques).

Galactosidase $\beta 1$ (GLB1) staining. Fresh tumor tissue was frozen in liquid nitrogen following resection and processed for embedding in O.C.T. compound. Tissue sections (5- $\mu$ m-thick) 
were collected and processed for GLB1 staining. GLB1 staining was performed using a Senescence $\beta$-Galactosidase Staining kit (cat. no. 9860, Cell Signaling Technology) according to the manufacturer's protocol. Micrographs were acquired under a light microscope (Nikon Eclipse Ti-U, Nikon Instruments). Positive results for GLB1 staining were calculated using Photoshop CS6 software (Adobe Systems).

Immunohistochemistry. Tumor tissue obtained from the animals was fixed in formalin for $24 \mathrm{~h}$ at room temperature and incubated in $70 \%$ ethanol for $48 \mathrm{~h}$ before being embedded in paraffin. The embedded tumors were cut into $5-\mu \mathrm{m}$-thick sections, mounted on poly-L-lysine-coated slides and then stained with hematoxylin and eosin (H\&E) to analyze the tumor histology. Immunohistochemical staining was carried out as previously described (9). Cell proliferation was analyzed using antibodies against Ki67 (Cell Signaling Technology, 1:400 dilution). Images were acquired with a CCD camera (Coolsnap ES, Roper Scientific) equipped with Metamorph software (Molecular Devices).

Statistical analysis. In this study, data were evaluated using SPSS version 22.0 statistical software (IBM Corp.) and analyzed using an unpaired two-tailed t-test or repeated measures analysis of variance. Differences were considered statistically significant at $\mathrm{P}<0.05$.

\section{Results}

AAV-NUPRI shRNA decreases NUPRI expression levels in A549 cells in vitro. To verify the effects of AAV-NUPRI shRNA on the expression of NUPR1, AAV-control (cont), 4, 20, 40 and $200 \mathrm{vg} / \mathrm{cell}$ of the AAV-based vector containing NUPR 1 shRNA were used to infect A549 cells. Western blot analysis demonstrated that AAV-NUPRl shRNA decreased the NUPR1 protein expression levels in A549 cells (Fig. 1A and B) in a dose-dependent manner. These data indicated that AAV-delivered shRNA significantly decreased NUPR1 protein expression levels in vitro.

AAV-NUPRI shRNA impairs the autophagic process and causes premature senescence in A549 cells. Since it has already been verified that the downregulation of NUPR1 impairs tumor cell autophagy (9), this study then examined the expression of synaptosome associated protein 25 (SNAP25), sequestosome 1 (SQSTM1/p62) and microtubule-associated protein 1 light chain 3B (MAP1LC3B/LC3B) in A549 cells by western blot analysis to confirm its biological effects. As expected, NUPRI depletion by the AAV system decreased the SNAP25 protein expression level and increased LC3B-I to LC3B-II conversion; all these effects were enhanced by treatment with Torin 1 (an mTOR inhibitor and autophagy inducer) (Fig. 2A), consistent with previous findings. We then examined key cell cycle inhibitors (p16, p21 and p27) by western blot analysis. AAV-mediated NUPR1 shRNA treatment resulted in the upregulation of $\mathrm{p} 21$ and $\mathrm{p} 27$ expression, but not p16 expression (Fig. 2B), which is a deleted mutation in A549 cells. As expected, AAV-mediated NUPRI depletion in A549 cells led to a significant increase in the number of GLB1-positive cells (Fig. 2C). These data indicated that AAV-mediated NUPRI

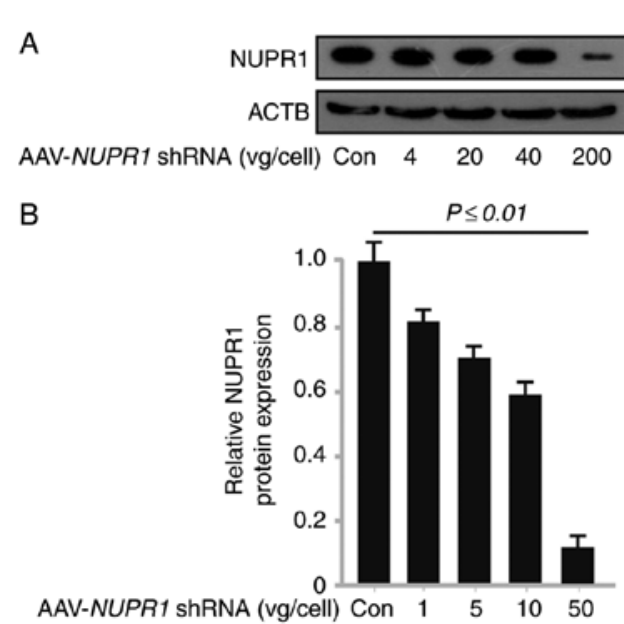

Figure 1. AAV-NUPR1 shRNA downregulates NUPR1 in A549 cells. (A) Western blot analysis of NUPR1 protein expression in A549 cells following treatment with AAV-control (cont), 4, 20,40 and $200 \mathrm{vg} / \mathrm{cell}$ of the AAV-based vector containing NUPRI shRNA for $48 \mathrm{~h}$. (B) The lower bar graph depicts the densitometric analysis of the protein intensity. NUPR1, nuclear protein-1; AAV, adeno-associated virus; ACTB, actin $\beta$.
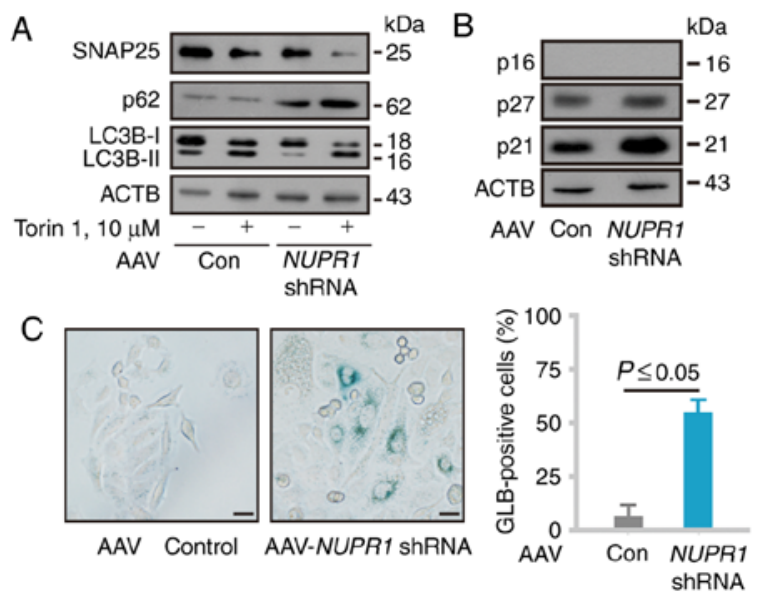

Figure 2. AAV-NUPRI shRNA impairs tumor cell autophagy and causes premature senescence. (A) Cells were sequentially infected with AAV-NUPRI shRNA and treated with $10 \mu \mathrm{M}$ Torin1. (B) Western blot analysis of the indicated proteins in A549 cells infected with AAV-NUPR1 shRNA using ACTB as a loading control. (C) Representative images of GLB1 activity in NUPR1-depleted cells mediated by AAV and in control cells (left panels). Quantification of GLB1-positive cells was conducted using 8 different fields of view from 3 independent experiments (mean \pm SEM) (right panel). Scale bars, $10 \mu \mathrm{m}$. NUPR1, nuclear protein-1; AAV, adeno-associated virus; SNAP25/CP13, synaptosome associated protein 25; SQSTM1/P62, nuclear pore glycoprotein $\mathrm{p} 62$; MAP1LC3B/LC3B, microtubule-associated protein 1 light chain $3 \mathrm{~B}$; $A C T B$, actin $\beta$; GLB1, galactosidase $\beta 1$.

shRNA impaired tumor cell autophagy, leading to premature senescence in vitro.

Combination of AAV-mediated NUPRI knockdown with TFP decreases A549 tumorigenesis in vivo by a single injection. TFP, an antipsychotic drug, has been shown to act as a potent NUPR1 inhibitor, exerting antitumor effects (29). Subsequently, in this study, the effect of AAV-mediated NUPRI shRNA combined with TFP was assessed on tumorigenesis in vivo, hypothesizing that TFP enhances antitumor activity by NUPRI 
A
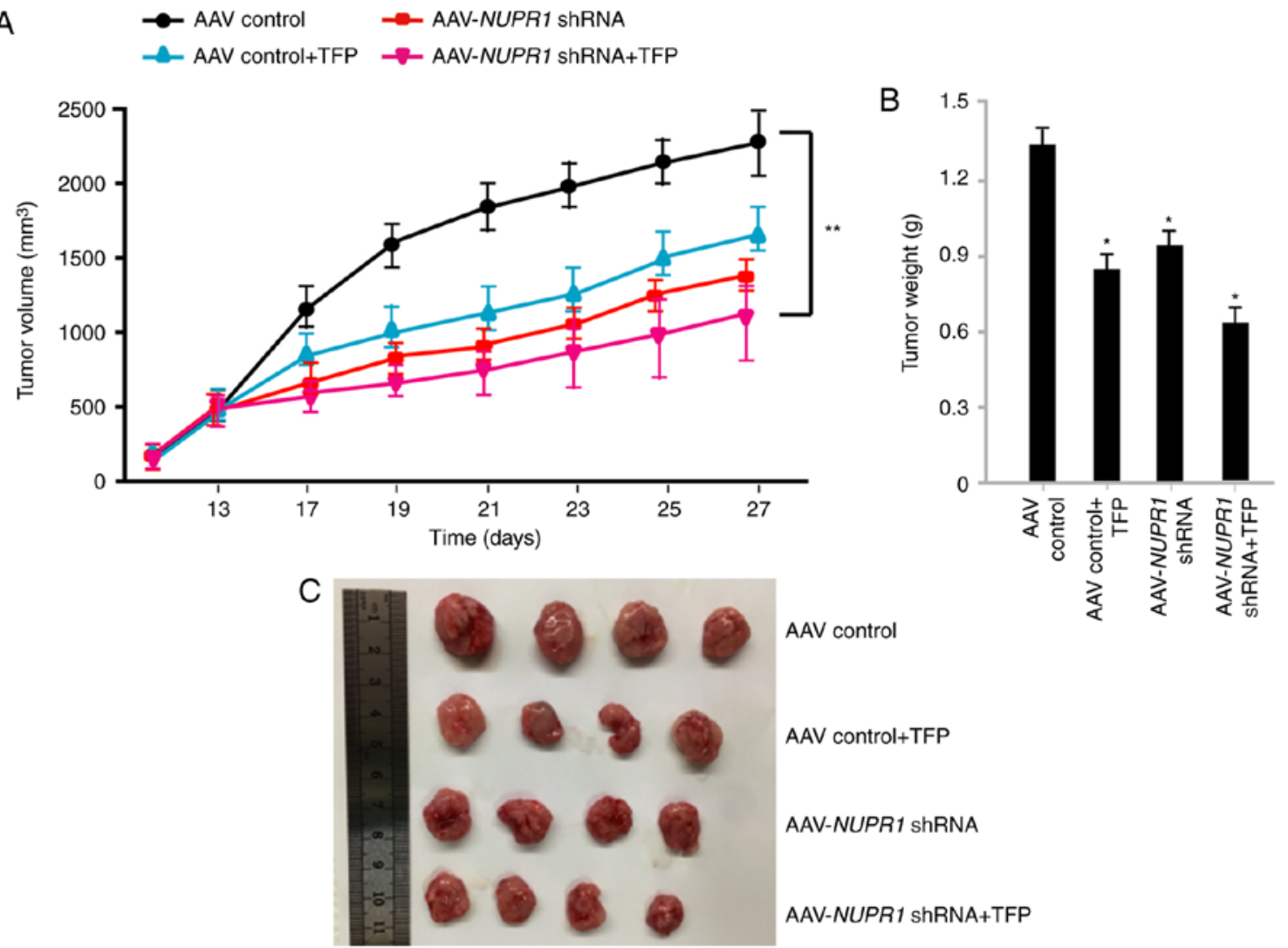

Figure 3. AAV-NUPR1 shRNA and TFP inhibits lung tumor growth. (A) Tumor growth in A549 tumor-bearing mice that were injected with TFP, AAV-NUPRI shRNA, AAV-NUPR1 shRNA and TFP, or AAV-control particles (n=4). (B) Tumors from mice treated with TFP, AAV-NUPR1 shRNA, AAV-NUPR1 shRNA and TFP, or AAV-control particles were weighed at the time of sacrifice $(n=4)$. The data are presented as the means \pm SD of 4 mice. ${ }^{*} \mathrm{P}<0.05$ and ${ }^{* *} \mathrm{P}<0.01$, compared to the control group. (C) Images of tumors at the experimental endpoint. NUPR1, nuclear protein-1; AAV, adeno-associated virus; TFP, trifluoperazine dihydrochloride.

depletion. A subcutaneous model was employed to analyze the application potential of TFP and AAV-NUPRI shRNA. A549 cells were subcutaneously injected into athymic nude mice. At 10 days following tumor cell inoculation, all the cells formed visible xenograft tumors. The mice were then randomly divided into four groups and received tail vein injections of AAV-NUPR1 shRNA, AAV-control (1 $11^{11} \mathrm{vg} /$ mouse), TFP, or saline as a control. The tumor volumes in the AAV-NUPRI shRNA/TFP-single-treated or double-treated groups were significantly smaller than those in the control group, and the tumor volumes of the double-treated group were the smallest among all the groups (Fig. 3A). Consistent with the tumor volume, the tumor weight and size in the AAV-NUPR1 shRNAand TFP-injected group were the most reduced compared with the other groups (Fig. 3B and C). There was no significant difference in tumor volume, weight and size between single therapy and combination therapy; however, combination therapy still exerted more beneficial tumor-suppressive effects, which was useful in reducing the dose of the NUPRI shRNA virus. Both the tumor volume and weight were significantly decreased by combination treatment (Fig. 3A-C).

$A A V$-mediated downregulation of NUPRI and TFP treatment causes autophagy dysregulation and premature senescence in vivo. To investigate whether the AAV-mediated downregulation of NUPRI has the same effect in vivo as in vitro, histological examinations confirmed the decrease in the tumor volume in the AAV-NUPRI shRNA- and TFP-injected groups, with a high cell death rate and substantially decreased numbers of scattered tumor cells (Fig. 4A). Evidence to support this phenomenon was also provided by transmission electron micrographs exhibiting significantly increased autolysosomal vacuole formation upon AAV-NUPRl shRNA and TFP treatment on day 27 (Fig. 4B). This phenomenon that has been previously described (9) is caused by impaired autolysosomal processing, which is not favorable in cancer cells. Immunohistochemical analyses confirmed that the NUPR1 expression level was decreased at 27 days following the injection of AAV-NUPRI shRNA or TFP, which was not observed in the control tumors. Moreover, significant decreases in the NUPR1 expression level and in the number of cells positive for Ki67 were observed, which is a marker of cell proliferation, in the AAV-NUPRI shRNA and TFP-treated groups compared with the control group (Fig. 4C); no significant difference was observed between single therapy and combination therapy. Subsequently, whether AAV-NUPR1 shRNA causes premature senescence in lung tumor xenografts was assessed. As shown in the data summary (Fig. 4D), the AAV-NUPR1 shRNA-treated group and the combined AAV-NUPRI shRNA- and-TFP treated group exhibited significantly more GLB1-stained cells than the other two groups, suggesting that AAV-NUPRI shRNA triggers premature senescence in xenograft lung tumors. It is noteworthy that the number of GLB-positive cells in the 

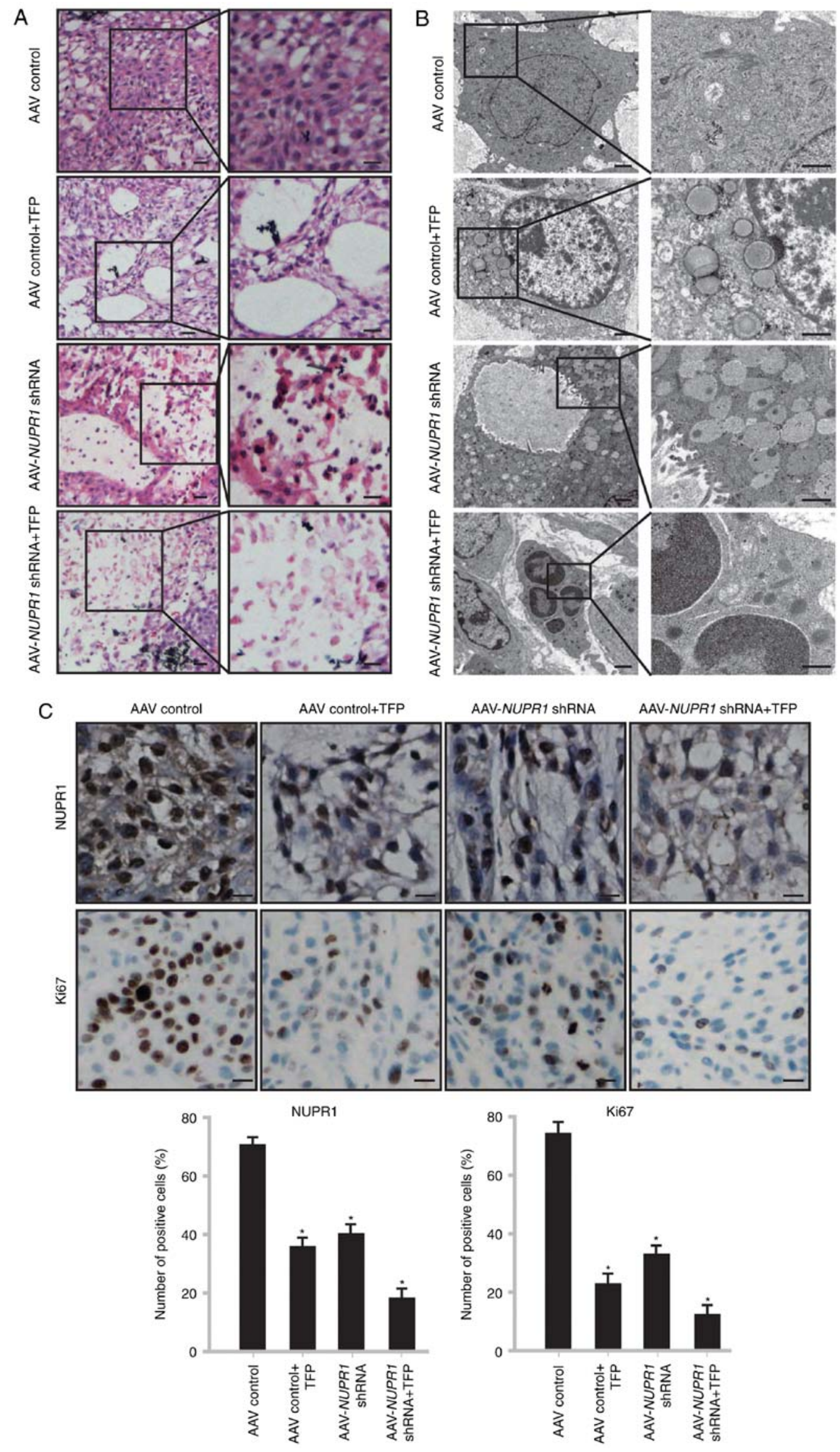

Figure 4. Effects of AAV-NUPR1 shRNA and TFP on A549 xenograft tumor. (A) Representative transverse sections of tumors from treated mice were stained with hematoxylin and eosin (H\&E). Scale bars, $100 \mu \mathrm{m}$. (B) A transmission electron micrograph of tumor tissue excised at 27 days after administering viral particles or a TFP injection. Scale bars, $200 \mathrm{~nm}$. (C) Sections of transplanted tumors infused with TFP, AAV-NUPR1 shRNA, AAV-NUPR1 shRNA and TFP, or AAV-control particles were stained with NUPR1 and Ki67 antibodies (upper panels). Intratumoral staining was quantified by the intensity multiplied by the percentage of positive cells (mean $\pm \mathrm{SD}$ ) (lower panels). ${ }^{*} \mathrm{P}<0.01$, compared to the AAV-control. Scale bars, $10 \mu \mathrm{m}$. 


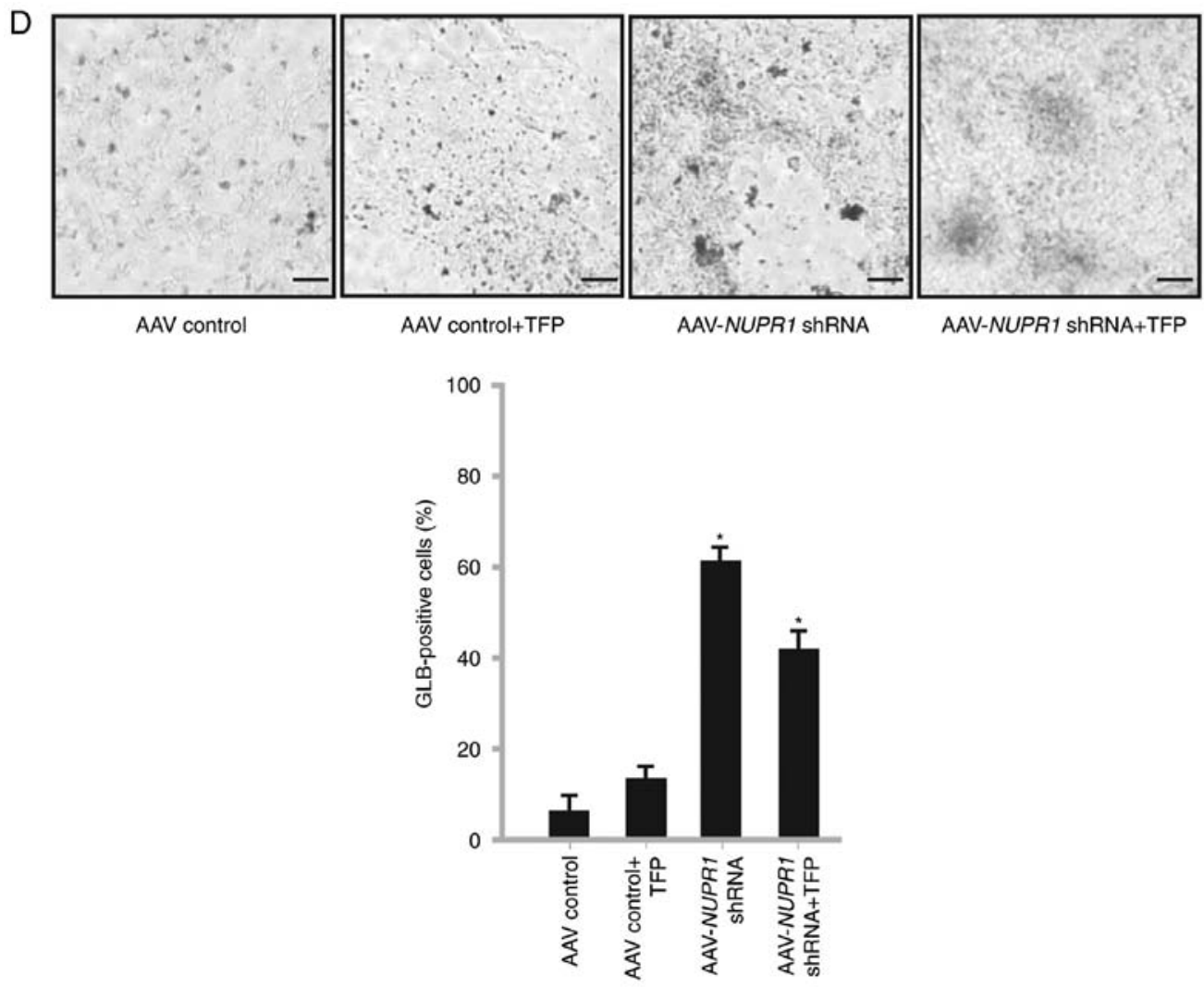

Figure 4. Continued. (D) Representative images of GLB1 activity in frozen sections of transplanted tumors infused with TFP, AAV-NUPR1 shRNA, AAV-NUPR1 shRNA and TFP, or AAV-control particles (upper panels). Quantification of GLB1-positive cells was conducted using 10 different fields of view from 3 independent experiments (mean \pm SEM) (lower panels). Scale bars, $50 \mu \mathrm{m}$. NUPR1, nuclear protein-1; AAV, adeno-associated virus; TFP, trifluoperazine dihydrochloride; GLB1, galactosidase $\beta 1$.

NUPR1 shRNA-treated group was increased compared to that in the combination therapy group. However, the tumor weight and size in the combination therapy group were more greatly reduced compared with those in the NUPRI shRNA-treated group, suggesting that TFP may mediate non-senescence cell death to impair lung tumor growth. The exact function of TFP in tumors is not yet clear. The results of this study indicated that it can interfere with the premature senescence mediated by NUPR1 shRNA treatment; however, this warrants further investigation. Overall, the results of this study indicated that AAV-NUPRI shRNA efficiently decreased A549 xenograft tumor cell proliferation, impaired autophagy, caused premature senescence and further inhibited the growth of tumor xenografts in nude mice; TFP enhanced all these effects, apart from premature senescence. The combination of AAV-NUPRI shRNA and TFP treatment is effective in inhibiting the growth of xenograft tumors.

\section{Discussion}

As a transcriptional co-regulator, the expression level of nuclear protein-1 (NUPR1) is relatively higher in the majority of non-small cell lung cancer (NSCLC) cell lines examined (9). In this study, it was found that adeno-associated virus (AAV)-NUPRI shRNA decreased NUPR1 expression levels in A549 cells in a dose-dependent manner. Moreover, NUPRI knockdown also impaired the autophagic process and caused premature senescence in A549 cells, consistent with the results of previous studies by the authors (9). Both AAV-NUPRI shRNA and trifluoperazine dihydrochloride (TFP) treatments decreased A549 tumorigenesis in vivo, and combined treatment enhanced the effects of single treatment using either AAV-NUPR1 shRNA or TFP. The in vitro experimental data of the autophagic process and premature senescence were reproduced in an in vivo mouse model using A549 cell lines with NUPR1 knockdown by AAV-NUPRI shRNA and/or treatment with TFP.

In a previous study by the authors, NUPRI depletion impaired autophagic flux dynamics and induced autolysosomal vacuolization and premature senescence via the downregulation of SNAP25 expression (9). In addition, NUPR1 is a stress-inducible transcriptional co-factor (30) that is specifically expressed in several types of cancer $(9,13,31)$ and participates in a number of cancer-associated processes (32-34). This suggests that NUPR1 plays an important role in promoting cancer development and progression without affecting, at least in part, adjacent normal tissues. Consistent with these results, the data of this study suggest that NUPR1 inhibition may be a novel therapeutic target for the treatment of NSCLC.

The findings of this study demonstrated that NUPRI knockdown by the AAV system resulted in impaired tumor cell autophagy, leading to premature senescence in vitro and delayed xenograft tumor growth in vivo, consistent with the results of a previous study by the authors using a lentiviral shRNA delivery system (9). To the best of our knowledge, this is the first report of the biological function of NUPRI 
knockdown by AAV-mediated shRNA delivery in NSCLC. The data indicate that AAV is a very effective tool for shRNA delivery and may be developed as a therapeutic strategy for targeting NUPRl in NSCLC. Although AAV is a potentially useful tool in the field of gene therapy and is less pathogenic than adenovirus (26), potential risks caused by AAV integration remain (35). Another major concern associated with the application of AAV-mediated NUPR1 shRNA delivery for the treatment of tumors in vivo is its large-scale production, since AAV production is time-consuming and costly. Therefore, it is necessary to improve AAV-mediated NUPRI shRNA therapy.

A number of clinical investigations have found that antipsychotic drugs, such as penfluridol, chlorpromazine and thioridazine may have potential anticancer potential effects for clinical treatment (36). TFP, which is a member of the phenothiazine class of antipsychotic drugs, has been evaluated in phase I and phase II clinical trials for the treatment of non-Hodgkin's lymphoma and glioblastoma multiforme, respectively $(37,38)$. TFP activates the intrinsic apoptotic pathway in TNBC cells (39) and A549 human lung cancer cells (21). Recently, a TFP-derived compound was produced and exhibited a dose-dependent tumor regression with no neurological effects and an ability to induce cell death mainly by necroptosis (29). Nevertheless, the present study demonstrated that TFP binding indeed promotes changes in the concentration and conformation of NUPR1, leading to beneficial tumor-suppressive effects. Additionally, it was demonstrated that the tumor growth inhibitory effect of AAV-mediated NUPR 1 shRNA delivery combined with TFP treatment was more effective than single treatment alone, although the difference was not highly significant. Thus, TFP can reduce the therapeutic dose of the NUPRI shRNA virus. Reducing the dose of the NUPR1 shRNA virus may also aid in the prevention of adverse side-effects such as transient increases in transaminases associated with increased AAV capsid-specific $\mathrm{T}$ cells and decreased circulating human factor IX levels. More importantly, the mice did not exhibit significant weight loss or other signs of toxicity during treatment, indicating the safety and efficiency of the combination of AAV-mediated NUPRI shRNA delivery with TFP as a strategy for cancer therapy. It was also noted that the combination therapy did not lead to the complete reduction of tumor growth and volume, as this therapy targets NUPR1; as, it cannot be confirmed that all lung cancer cells express and are dependent on NUPR1, the effectiveness of the combination therapy is limited to NUPR1-positive lung cancer cells. Thus, the combination of TFP with AAV-mediated NUPRI shRNA delivery may prolong progression-free survival and achieve a better treatment response in patients with NUPR1-positive tumors. Since TFP can bind NUPR1, it may be predicted that radiolabeled TFP by fluorine-18 may be used as a potential positron-emitting imaging agent, as well as an antitumor drug for NUPR1-positive tumors.

In conclusion, to the best of our knowledge, this is the first study on AAV-mediated NUPRI shRNA delivery combined with treatment with TFP for NUPR1-positive tumors. It was demonstrated that the tail vein injection of AAV-mediated NUPRl shRNA with TFP significantly attenuated the growth of lung cancer cell xenografts. These findings suggest that
TFP combined with AAV-mediated NUPRI shRNA is a feasible and potential antitumor approach for preclinical studies.

\section{Acknowledgements}

The authors would like to acknowledge the laboratory equipment and platform support sponsored by the Tianjin Key Laboratory of Medical Epigenetics, Key Laboratory of Immune Microenvironment and Disease (Ministry of Education).

\section{Funding}

This study was supported by the National Natural Science Foundation of China (grant nos. 2018YFC1313002, 81825017, $81773034,31600636,81702245,81874039,81872350$ and 81572271), the Tianjin Municipal Science and Technology Commission (grant nos. 16JCYBJC24500, 18JCZDJC99100 and 19JCZDJC35600), and the open fund of the State Key Laboratory of Medicinal Chemical Biology (Nankai University) (grant no. 201501002).

\section{Availability of data and materials}

The datasets analyzed during the present study are available from the corresponding author on reasonable request.

\section{Authors' contributions}

ZL and ZM designed this study. YL, YY, JM, YS, RZ, BC, YZ and XY performed the experiments. YL and JY analyzed the data and prepared the figures. YL wrote the manuscript and XY revised the manuscript. All the authors have read and approved the final version of the manuscript for publication and agree to be accountable for all aspects of the research in ensuring that the accuracy or integrity of any part of the work are appropriately investigated and resolved.

\section{Ethics approval and consent to participate}

Animal care and surgical procedures were approved by Tianjin Medical University and carried out in accordance with the Institutional Animal Care and Use Committee guidelines (Tianjin Medical University, permission no. SYXK: 2016-0012).

\section{Patient consent for publication}

Not applicable.

\section{Competing interests}

The authors declare that they have no competing interests.

\section{References}

1. Hirsch FR, Scagliotti GV, Mulshine JL, Kwon R, Curran WJ Jr, Wu YL and Paz-Ares L: Lung cancer: Current therapies and new targeted treatments. Lancet 389: 299-311, 2017.

2. Herbst RS, Heymach JV and Lippman SM: Lung cancer. N Engl J Med 359: 1367-1380, 2008. 
3. Siegel R, Naishadham D and Jemal A: Cancer statistics, 2012. CA Cancer J Clin 62: 10-29, 2012.

4. Goruppi S, Patten RD, Force T and Kyriakis JM: Helix-loop-helix protein $\mathrm{p} 8$, a transcriptional regulator required for cardiomyocyte hypertrophy and cardiac fibroblast matrix metalloprotease induction. Mol Cell Biol 27: 993-1006, 2007.

5. Mallo GV, Fiedler F, Calvo EL, Ortiz EM, Vasseur S, Keim V, Morisset $\mathbf{J}$ and Iovanna JL: Cloning and expression of the rat $\mathrm{p} 8$ cDNA, a new gene activated in pancreas during the acute phase of pancreatitis, pancreatic development, and regeneration, and which promotes cellular growth. J Biol Chem 272: 32360-32369, 1997.

6. Jiang YF, Vaccaro MI, Fiedler F, Calvo EL and Iovanna JL: Lipopolysaccharides induce p8 mRNA expression in vivo and in vitro. Biochem Biophys Res Commun 260: 686-690, 1999.

7. Taïeb D, Malicet C, Garcia S, Rocchi P, Arnaud C, Dagorn JC, Iovanna JL and Vasseur S: Inactivation of stress protein $\mathrm{p} 8$ increases murine carbon tetrachloride hepatotoxicity via preserved CYP2E1 activity. Hepatology 42: 176-182, 2005.

8. Zinke I, Schütz CS, Katzenberger JD, Bauer M and Pankratz MJ: Nutrient control of gene expression in Drosophila: Microarray analysis of starvation and sugar-dependent response. EMBO J 21: 6162-6173, 2002

9. Mu Y, Yan X, Li D, Zhao D, Wang L, Wang X, Gao D, Yang J, Zhang H, Li Y, et al: NUPR1 maintains autolysosomal efflux by activating SNAP25 transcription in cancer cells. Autophagy 14 654-670, 2018.

10. Jung $\mathrm{SH}$, Lee A, Yim $\mathrm{SH}, \mathrm{Hu} \mathrm{HJ}$, Choe $\mathrm{C}$ and Chung YJ: Simultaneous copy number gains of NUPR1 and ERBB2 predicting poor prognosis in early-stage breast cancer. BMC Cancer 12: 382, 2012

11. Iovanna JL: Expression of the stress-associated protein $\mathrm{p} 8$ is a requisite for tumor development. Int J Gastrointest Cancer 31: 89-98, 2002.

12. Vasseur S, Hoffmeister A, Garcia S, Bagnis C, Dagorn JC and Iovanna JL: p8 is critical for tumour development induced by rasV12 mutated protein and E1A oncogene. EMBO Rep 3: 165-170, 2002.

13. Ito $\mathrm{Y}$, Yoshida $\mathrm{H}$, Motoo $\mathrm{Y}$, Iovanna JL, Tomoda C, Uruno $\mathrm{T}$, Takamura Y, Miya A, Kobayashi K, Matsuzuka F, et al: Expression of p8 protein in medullary thyroid carcinoma. Anticancer Res 25: 3419-3423, 2005.

14. Su SB, Motoo Y, Iovanna JL, Xie MJ and Sawabu N: Effect of camostat mesilate on the expression of pancreatitis-associated protein (PAP), p8, and cytokines in rat spontaneous chronic pancreatitis. Pancreas 23: 134-140, 2001.

15. Guo X, Wang W, Hu J, Feng K, Pan Y, Zhang L and Feng Y: Lentivirus-mediated RNAi knockdown of NUPR1 inhibits human nonsmall cell lung cancer growth in vitro and in vivo. Anat Rec (Hoboken) 295: 2114-2121, 2012

16. Vasseur S, Vidal Mallo G, Fiedler F, Bödeker H, Cánepa E, Moreno S and Iovanna JL: Cloning and expression of the human p8, a nuclear protein with mitogenic activity. Eur J Biochem 259: 670-675, 1999

17. Hamidi T, Algül H, Cano CE, Sandi MJ, Molejon MI, Riemann M, Calvo EL, Lomberk G, Dagorn JC, Weih F, et al: Nuclear protein 1 promotes pancreatic cancer development and protects cells from stress by inhibiting apoptosis. J Clin Invest 122: 2092-2103, 2012.

18. Santofimia-Castaño P, Rizzuti B, Abián O, Velázquez-Campoy A, Iovanna JL and Neira JL: Amphipathic helical peptides hamper protein-protein interactions of the intrinsically disordered chromatin nuclear protein 1 (NUPR1). Biochim Biophys Acta Gen Subj 1862: 1283-1295, 2018.

19. Neira JL, Bintz J, Arruebo M, Rizzuti B, Bonacci T, Vega S, Lanas A, Velázquez-Campoy A, Iovanna JL and Abián O Identification of a drug targeting an intrinsically disordered protein involved in pancreatic adenocarcinoma. Sci Rep 7: 39732, 2017.

20. Zhang L, Yu J, Pan H, Hu P, Hao Y, Cai W, Zhu H, Yu AD, Xie X, Ma D and Yuan J: Small molecule regulators of autophagy identified by an image-based high-throughput screen. Proc Natl Acad Sci USA 104: 19023-19028, 2007.
21. Chen QY, Wu LJ, Wu YQ, Lu GH, Jiang ZY, Zhan JW, Jie Y and Zhou JY: Molecular mechanism of trifluoperazine induces apoptosis in human A549 lung adenocarcinoma cell lines. Mol Med Rep 2: 811-817, 2009.

22. Annabi B, Pilorget A, Bousquet-Gagnon N, Gingras D and Béliveau R: Calmodulin inhibitors trigger the proteolytic processing of membrane type-1 matrix metalloproteinase, but not its shedding in glioblastoma cells. Biochem J 359: 325-333, 2001

23. Chen MH, Lin KJ, Yang WLR, Kao YW, Chen TW, Chao SC, Chang PM, Liu CY, Tzeng CH, Chao Y, et al: Gene expression-based chemical genomics identifies heat-shock protein 90 inhibitors as potential therapeutic drugs in cholangiocarcinoma. Cancer 119: 293-303, 2013

24. Shin SY, Kim CG, Hong DD, Kim JH and Lee YH: Implication of Egr-1 in trifluoperazine-induced growth inhibition in human U87MG glioma cells. Exp Mol Med 36: 380-386, 2004.

25. Yeh CT, Wu ATH, Chang PMH, Chen KY, Yang CN, Yang SC, Ho CC, Chen CC, Kuo YL, Lee PY, et al: Trifluoperazine, an antipsychotic agent, inhibits cancer stem cell growth and overcomes drug resistance of lung cancer. Am J Respir Crit Care Med 186: 1180-1188, 2012.

26. Miller N: Glybera and the future of gene therapy in the European Union. Nat Rev Drug Discov 11: 419, 2012.

27. Shi J, Zheng D, Liu Y, Sham MH, Tam P, Farzaneh F and Xu R: Overexpression of soluble TRAIL induces apoptosis in human lung adenocarcinoma and inhibits growth of tumor xenografts in nude mice. Cancer Res 65: 1687-1692, 2005.

28. Xiao W, Chirmule N, Schnell MA, Tazelaar J, Hughes JV and Wilson JM: Route of administration determines induction of T-cell-independent humoral responses to adeno-associated virus vectors. Mol Ther 1: 323-329, 2000.

29. Santofimia-Castaño P, Xia Y, Lan W, Zhou Z, Huang C, Peng L, Soubeyran P, Velázquez-Campoy A, Abián O, Rizzuti B, et al: Ligand-based design identifies a potent NUPR1 inhibitor exerting anticancer activity via necroptosis. J Clin Invest 129: 2500-2513, 2019

30. Goruppi S and Iovanna JL: Stress-inducible protein $\mathrm{p} 8$ is involved in several physiological and pathological processes. J Biol Chem 285: 1577-1581, 2010.

31. Li J, Ren S, Liu Y, Lian Z, Dong B, Yao Y and Xu Y: Knockdown of NUPR1 inhibits the proliferation of glioblastoma cells via ERK1/2, p38 MAPK and caspase-3. J Neurooncol 132: 15-26, 2017.

32. Gironella M, Malicet C, Cano C, Sandi MJ, Hamidi T, Tauil RMN, Baston M, Valaco P, Moreno S, Lopez F, et al: p8/nupr1 regulates DNA-repair activity after double-strand gamma irradiation-induced DNA damage. J Cell Physiol 221: 594-602, 2009.

33. Malicet C, Dagorn JC, Neira JL and Iovanna JL: p8 and prothymosin alpha: Unity is strength. Cell Cycle 5: 829-830, 2006.

34. Sandi MJ, Hamidi T, Malicet C, Cano C, Loncle C, Pierres A, Dagorn JC and Iovanna JL: p8 expression controls pancreatic cancer cell migration, invasion, adhesion, and tumorigenesis. J Cell Physiol 226: 3442-3451, 2011.

35. Chandler RJ, Sands MS and Venditti CP: Recombinant adenoassociated viral integration and genotoxicity: Insights from animal models. Hum Gene Ther 28: 314-322, 2017.

36. Wu L, Liu YY, Li ZX, Zhao Q, Wang X, Yu Y, Wang YY, Wang YQ and Luo F: Anti-tumor effects of penfluridol through dysregulation of cholesterol homeostasis. Asian Pac J Cancer Prev 15: 489-494, 2014

37. Hait WN, Byrne TN, Piepmeier J, Durivage HJ, Choudhury S, Davis CA and Gates JA: The effect of calmodulin inhibitors with bleomycin on the treatment of patients with high grade gliomas. Cancer Res 50: 6636-6640, 1990.

38. Hait WN, Morris S, Lazo JS, Figlin RJ, Durivage HJ, White K and Schwartz PE: Phase I trial of combined therapy with bleomycin and the calmodulin antagonist, trifluoperazine. Cancer Chemother Pharmacol 23: 358-362, 1989.

39. Feng Z, Xia Y, Gao T, Xu F, Lei Q, Peng C, Yang Y, Xue Q, Hu X, Wang $\mathrm{Q}$, et al: The antipsychotic agent trifluoperazine hydrochloride suppresses triple-negative breast cancer tumor growth and brain metastasis by inducing G0/G1 arrest and apoptosis. Cell Death Dis 9: 1006, 2018. 